\title{
PUVA versus NB-UVB in Management of Vitiligo, Clinico-Immuno-Pathological Study
}

\author{
Sameh K. Attia, Sherif S. Awad, Siham Yacoub \\ Department of Dermatology and Venereology, Minia University, Minya, Egypt. \\ Email: sherifu@rocketmail.com
}

Received September $3^{\text {rd }}, 2013$; revised October $1^{\text {st }}, 2013$; accepted October $9^{\text {th }}, 2013$

Copyright (c) 2013 Sameh K. Attia et al. This is an open access article distributed under the Creative Commons Attribution License, which permits unrestricted use, distribution, and reproduction in any medium, provided the original work is properly cited.

\begin{abstract}
Background: Phototherapy is the most commonly used modality in the treatment of vitiligo. Oral PUVA is the classical treatment and the NB-UVB is a recently introduced form excluding the shorter erythemogenic wavelengths. Aims: This study was designed to compare the effects of PUVA and NB-UVB clinically and immuno-pathologically in managing non segmental vitiligo. Patients/Methods: Thirty vitiligo patients were divided randomly into two groups and treated either by oral PUVA or by narrow band UVB for 4 months, and evaluation was done clinically and immuno-pathologically. Results: One patient in PUVA group (3.3\%) failed to respond to therapy while 29 patients (93.3\%) improved including all NB-UVB cases. Excellent repigmentation was achieved in 6.7\% in PUVA group and 66.6\% in NB-UVB group; good repigmentation was achieved in 60\% in PUVA and 20\% in NB-UVB while 26.7\% in PUVA and 13.3\% in NB-UVB showed mild repigmentation. The color matching was excellent in all NB-UVB patients. Recurrence and activation of vitiligo were demonstrated in some NB-UVB cases and were less in PUVA treated cases. Microscopic examination revealed persistence of dermal lympho-histiocytic infiltrate and the interface changes in biopsies from vitiliginous lesions treated with NB-UVB more than with PUVA. Conclusions: NB-UVB provides significant better results than oral PUVA in managing non-segmental vitiligo. Although NB-UVB therapy gives a rapid effect, yet the observation of recurrences and development of new lesions of vitiligo were less significant with PUVA. It was also observed that PUVA has better immuno-modulatory effect on vitiligo than NB-UVB and may give better response on a longer period of time.
\end{abstract}

Keywords: PUVA; Vitiligo; NB-UVB

\section{Introduction}

Vitiligo is an acquired pigmentary disorder characterized by loss of melanocytes from the skin and subsequent development of depigmented patches of variable sizes, which may enlarge and coalesce to form extensive areas of leukoderma [1].

Treatment modalities aim to stimulate melanocyte proliferation or interfere with inflammatory factors affecting melanocyte structure or function; however, no single treatment method has been found to be consistently effective, with relatively few side effects [2]. Ultraviolet irradiation is among the many possible approaches and is divided into ultraviolet A (UVA) (320 - $400 \mathrm{~nm})$, ultraviolet B (UVB) (290 - $320 \mathrm{~nm}$ ) and ultraviolet C (UVC) (200 - $290 \mathrm{~nm}$ ) [3]. PUVA (psoralen followed by irradiation with UVA) has been a well established management of non-segmental vitiligo since the last century [4]. Nar- row-band UV-B therapy was introduced recently as new light therapy which emits a concentrated UVB source of $311 \mathrm{~nm}$ and has been shown to have a profound therapeutic efficacy for the treatment of skin conditions including vitiligo [5].

The aim of this work is to study and to compare the effects of PUVA versus narrow band UVB (NB-UVB) treatment on vitiligo patients clinically and immunopathologically.

\section{Patients and Methods}

This study was conducted on 30 vitiligo patients attending the out-patient clinic of Dermatology \& Venereology Department, Minia University Hospital after obtaining their consent and approval of the local ethical committee. These patients were divided randomly into two groups: 15 patients were treated by PUVA and 15 patients were 
treated by NB-UVB.

Vitiligo patients included in the study had non segmental vitiligo in generalized or localized forms. Exclusion criteria enclosed: patients receiving treatment in the last 2 months, patients with light sensitive or aggravated dermatoses, patients with or giving past history of skin tumors, patients with aphakia or cataract. PUVA treatment group also excluded children, pregnant or lactating female patients and patients with abnormal liver or renal functions.

All patients were subjected to full history taking including, general clinical examination including: abdominal, chest, cardiac, ophthalmological and ENT examination, dermatological examination including the type and extent of vitiligo and complete routine laboratory investigations, including complete blood picture and liver and kidney function tests. Repeated laboratory tests were performed every two months after starting treatment in patients who were treated by PUVA.

In PUVA group, the patient ingested 8-MOP at a dose of $0.4 \mathrm{mg} / \mathrm{kg}$ of body weight $/ \mathrm{session}$ on a full stomach 1 and half hour before UVA exposure. The UVA cabinet used was UV compact irradiation cabinet COSMEDICO Medizintechnik, GP-42, Germany. Patients received 2 PUVA sessions/week. The starting dose was $2 \mathrm{~J} / \mathrm{cm}^{2}$, which is a common starting dose for patients in similar skin type [6] and 25\% dose increment was provided every next session [7] guided by the patients response till asymptomatic erythema was observed. Erythema reading for PUVA treated patients was performed at 72 hours after UVA irradiation [6]. The last previous reading was utilized as the therapeutic dose throughout the 4 months of therapy. Patients were instructed to wear protective goggles during UVA exposure and sunglasses for 12 hours after the session. The genital area was shielded in all cases.

In NB-UVB group, patients received 2 NB-UVB sessions/week. The starting dose was $0.574 \mathrm{~J} / \mathrm{cm}^{2}$, which is the starting dose of skin type IV, according to manufacture instructions and 25\% dose increment was done every next session guided by the patients' response, till asymptomatic erythema was observed. Erythema reading for NB-UVB treated patients was performed at 24 hours after UVB irradiation [8], the previous dose was utilized as the therapeutic dose throughout the 4 months of therapy. NB-UVB phototherapy cabinet used was COSMEDICO Medizintechnik, GH-8 ST, Germany, containing fluorescent TL-01 $(100 \mathrm{~W})$ tubes as the source of irradiation.

Every patient was photographed before the start of treatment then repeatedly every two months throughout PUVA and NB-UVB treatment.

Skin biopsies were harvested from each patient before PUVA and NB-UVB therapy (1 biopsy from the center of the vitiliginous area) and after 4 months of PUVA and
NB-UVB therapy (2 biopsies; one from the non-responding areas and the other biopsy from the repigmented areas). Three and half millimeter punch skin biopsies were used to obtain all samples after Mepivacaine HCL 3\% local anesthesia. Biopsies were fixed in $10 \%$ formalin and embedded in paraffin blocks and sectioned by microtome into $5 \mu \mathrm{m}$ thick sections. The resulting sections were mounted on glass slides and subjected to histopathological examination using Hematoxyline-Eosin, Fontana Masson and immunohistochemical staining using melanoma antigen recognized by T-lymphocyte (MART-1), CD3, CD20 and CD68.

MART-1 antibody (1:100, Neomarker, Fremont, CA 94539, USA) recognizes an $18 \mathrm{KD}$ molecular weight MART-1 antigen which is present in endoplasmic reticulum of melanocytes. It has been found to localize to melanosomes suggesting a role in melanosomes biogenesis and it is useful for biological studies on melanocytes and melanoma cells as well as for the development and monitoring of immunotherapy for the patients with melanoma [9]. CD3 marker (1:200, Dako, code No./M 7254) is a Pan-T lymphocyte marker. It recognizes CD3 antigen which is a pan- $\mathrm{T}$ cell antigen, composed of 5 invariable polypeptide chains. CD20 marker (1:400, Dako, code No./M 0755) is a pan-B lymphocyte marker. It recognizes $\mathrm{CD} 20$ antigen which is present in the $\mathrm{B}$ lymphocyte. CD68 marker (1:300, Neomarkers Fremont, CA, Lot: 397P601A) recognizes CD68 antigen which is present in the cytoplasm of histiocytes. This marker is important for identifying histiocytes in tissue sections.

After 2 and 4 months of therapy, we evaluated the patients subjectively into improvement or failure. The patients who showed improvement were evaluated objectively by using an instrument called planimeter. The improved patients were divided into three groups; excellent group of patients when improvement ranged between $70 \%-100 \%$, good repigmentation group of patients when improvement ranged between $40 \%$ \& $70 \%$, and mild repigmentation group of patients when improvement is less than $40 \%$. Planimeter is a measuring instrument used to measure the area of an irregular plane figure on a map or photograph. A pointer on the planimeter is used to trace around the boundary of the shape. This induces a movement in another part of the instrument and a reading of this is used to establish the area of the figure.

The resulting data were analyzed with SPSS statistical package version 12.0 (SPSS Inc., Chicago) for windows (Microsoft Corporation, Redmond). Data were expressed as mean \pm standard deviation. Comparison between groups of data was done by independent samples (unpaired) t-test or ANOVA (between 2 or more groups). Comparison between baseline data and after treatment was done by paired t-test. The difference between compared groups was expressed as probability of value ( $p$ value). 
The difference was considered significant if $p<0.05$.

\section{Results}

The present study was conducted on 27 female patients (90\%) and 3 male patients (10\%). The age of these patients at the time of examination ranged from 7 to 52 years old. The duration of the disease prior to phototherapy ranged from 2 months to 8 years. A positive family history of vitiligo was present in 5 (16.6\%). Regarding skin Fitzpatrick types; 2 patients (6.6\%) were skin type III, 20 patients (66.7\%) were skin type IV, 8 patients (26.7\%) were skin type $\mathrm{V}$.

In PUVA treated patients, 13 female patients (86.7\%) and 2 male patients (13.3\%) were included. The age of these patients at the time of examination ranged from 21 to 52 years, with a mean of $29.8( \pm 12.09)$ years. The duration of the disease prior to PUVA ranged from 2 months to 8 years, with a mean of 37.73 months \pm 29 SD. A positive family history of vitiligo was present in 4 patients $(26.7 \%)$. The erythemogenic dose ranged from 4.883 to $7.629 \mathrm{~J} / \mathrm{cm}^{2}$ and the therapeutic dose of PUVA ranged from 3.906 to $6.104 \mathrm{~J} / \mathrm{cm}^{2}$ (Table 1). As regard skin type, 1 patient (6.7\%) was skin type III, 11 patients (73.3\%) were skin type IV and 3 patients (20\%) were skin type V (Table 1).

In NB-UVB treated patients, 14 female patients (93.3\%) and 1 male patient (6.7\%) were in this study. The age of these patients at the time of examination ranged from 7 to 45 years with a mean of 16.2 years \pm $11.02 \mathrm{SD}$. The duration of the disease prior to NB-UVB ranged from 2 months to 8 years with a mean of 30.53 months \pm 26.27 SD. A positive family history of vitiligo was present in 1 patient (6.7\%). The erythemogenic dose ranged from 1.752 to $3.422 \mathrm{~J} / \mathrm{cm}^{2}$ and the therapeutic dose of UVB ranged from 1.402 to $2.737 \mathrm{~J} / \mathrm{cm}^{2}$ (Table 1). Regarding skin type, 1 patient (6.7\%) was skin type III, 9 patients (60\%) were skin type IV, and 5 patients (33.3\%) were skin type $\mathrm{V}$ (Table 1).

At the end of the second month of PUVA therapy improvement of the lesions occurred in 14 patients while 1 patient did not improve at all. Improvement ranged from $6 \%$ to $37 \%$ with a mean of $17.13 \pm 10.08$ SD.

At the end of the fourth month of therapy 1 patient (3.3\%) failed to respond to therapy and the 14 patients (93.3\%) showed improvement which ranged from 10\% to $70 \%$ with a mean of $39.27 \pm 18.41 \mathrm{SD}$ (Table 2).

At the end of the second month of NB-UVB therapy all 15 patients showed improvement ranging from $12 \%$ to $90 \%$ with a mean of $45.4 \pm 21.57$ SD. This difference in the range of improvement between the two study groups (PUVA and NB-UVB) after 2 months therapy was statistically significant; $p$ value $=0.0005$.

At the end of the fourth month of therapy all 15 patients showed improvement which ranged from 23\% to
Table 1. Data of the patients included in each group.

\begin{tabular}{|c|c|c|}
\hline Items & PUVA & NB-UVB \\
\hline $\begin{array}{l}\text { Age of patients at the } \\
\text { start of treatment }\end{array}$ & $\begin{array}{c}21 \text { to } 52 \text { yrs } \\
\text { Mean of } 29.8 \pm 12.09\end{array}$ & $\begin{array}{c}7 \text { to } 45 \mathrm{yrs} \\
\text { Mean of } 16.2 \pm 11.02\end{array}$ \\
\hline Sex & $\begin{array}{l}13 \text { females }(86.7 \%) \\
\text { and } 2 \text { males }(13.3 \%)\end{array}$ & $\begin{array}{l}14 \text { females }(93.3 \%) \\
\text { and } 1 \text { male }(6.7 \%)\end{array}$ \\
\hline Family history & 4 patients (26.7\%) & 1 patient $(6.7 \%)$ \\
\hline Skin type & $\begin{array}{c}\text { Type III: } 1 \text { (6.7\%) } \\
\text { Type IV: } 11 \text { (73.3\%) } \\
\text { Type V: } 3(20 \%)\end{array}$ & $\begin{array}{c}\text { Type III: } 1 \text { (6.7\%) } \\
\text { Type IV: } 9 \text { (60\%) } \\
\text { Type V: } 5 \text { (33.3\%) }\end{array}$ \\
\hline $\begin{array}{l}\text { Duration of vitiligo } \\
\text { prior to treatment }\end{array}$ & $\begin{array}{c}2 \mathrm{~ms} \text { to } 8 \text { yrs } \\
\text { Mean of } 37.73 \pm 29\end{array}$ & $\begin{array}{c}2 \text { ms to } 8 \text { yrs } \\
\text { Mean of } 30.53 \pm 26.27\end{array}$ \\
\hline The therapeutic dose & 3.906 to $6.104 \mathrm{~J} / \mathrm{cm}^{2}$ & 1.402 to $2.737 \mathrm{~J} / \mathrm{cm}^{2}$ \\
\hline
\end{tabular}

Table 2. Percentage of improvement after 2 and 4 months.

\begin{tabular}{lcccc}
\hline \multirow{2}{*}{ Percentage of improvement } & \multicolumn{4}{c}{ Study groups } \\
\cline { 3 - 5 } & & NB-UVB & PUVA & $p$-value* \\
\hline $\begin{array}{l}\text { Percentage of } \\
\text { improvement }\end{array}$ & Range & $12-90$ & $6-37$ & \\
after 2 months & Mean \pm SD & $45.4 \pm 21.57$ & $17.13 \pm 10.08$ & 0.0005 \\
& $p$-value & - & - & - \\
Percentage of & Range & $23-98$ & $10-70$ & \\
$\begin{array}{l}\text { improvement } \\
\text { after 4 months }\end{array}$ & Mean \pm SD & $73.27 \pm 24.94$ & $39.27 \pm 18.41$ & 0.0002 \\
& $p$-value & 0.0001 & 0.0005 & - \\
\hline
\end{tabular}

*Comparison between NB-UVB and PUVA groups; ${ }^{* *}$ Comparison between $\%$ of improvement after 2 months and after 4 months.

$98 \%$ with a mean of $73.27 \pm 24.94$ SD. This difference in the range of improvement between the two study groups (PUVA and NB-UVB) after 4 months therapy was statistically significant; $p$ value $=0.0002$ (Table 2 ).

Improved patients were divided into three groups (Table 3):

Excellent repigmentation (70\% - 100\%): in 1 patient (6.7\%) in PUVA group and in 10 patients $(66.7 \%)$ in NB-UVB group.

Good repigmentation (40\% - 70\%): in 9 patients (60\%) in PUVA group and in 3 patients (20\%) in NB-UVB group.

Mild repigmentation (less than 40\%): in 4 patients (26.7\%) in PUVA group and in 2 patients (13.3\%) in NB-UVB group.

The perifollicular repigmentation was the predominant type of repigmentation observed in both PUVA and NBUVB treated groups. It occurred in all improved patients $(100 \%)$ who were treated either by PUVA or NB-UVB. Only 1 patient (6.7\%), who was treated with NB-UVB, showed combination of both perifollicular repigmentation and peripheral repigmentation. Diffuse repigmentation was not observed in any patient.

The assessment of color match in comparison with the 
Table 3. Degree of repigmentation after treatment.

\begin{tabular}{ccc}
\hline $\begin{array}{c}\text { Degree of } \\
\text { repigmentation }\end{array}$ & $\begin{array}{c}\text { PUVA group } \\
\mathrm{N}=14\end{array}$ & $\begin{array}{c}\text { NB-UVB group } \\
\mathrm{N}=15\end{array}$ \\
\hline Excellent repigmentation & 1 patient (6.7\%) & 10 patients (66.7\%) \\
Good repigmentation & 9 patients (60\%) & 3 patients (20\%) \\
Mild repigmentation & 4 patients (26.7\%) & 2 patients (13.3\%) \\
\hline
\end{tabular}

patient's unaffected skin was excellent for all 15 patients $(100 \%)$ in the NB-UVB group and for only 8 patients (57.2\%) out of the 14 improved patients in the PUVA group while the remaining 6 patients (42.8\%) showed dark hyperpigmentation of the treated vitiliginous area than the patient's unaffected skin.

The side effects were minimal and did not warrant discontinuation of the therapy in any of the 30 patients. All patients in each group developed erythema, showing that both regimens were equally erythemogenic. Pruritus occurred in 4 patients $(26.7 \%)$ in the PUVA group compared with 3 patients (20\%) in the NB-UVB group. Photodermatitis with scaliness and eczematous reaction was observed only in 1 patient (6.7\%) who was treated with NB-UVB.

Throughout the study, recurrence of the vitiliginous patches at the same site of treatment occurred in 1 patient (6.7\%) in the PUVA group compared with 3 patients $(20 \%)$ in the NB-UVB group. New lesions developed in other sites in 1 patient (6.7\%) in the PUVA group compared with 4 patients (26.7\%) in the NB-UVB group.

Seven patients (46.7\%) who were treated with PUVA, developed nausea during the course of therapy and minimization of the dose of 8-MOP in those patients was performed to decrease the sensation of nausea. One female patient (6.7\%) who was treated with PUVA therapy developed elevation in the liver enzymes after 4 months of treatment and stopped the therapy (Table 4).

\section{Histopathological Findings}

Before treatment (Table 5, Figure 1), microscopic examination of involved skin in vitiligo showed no basal melanization in $22(73.3 \%)$ of the 30 biopsies while 8 (26.7\%) showed basal melanization. With FontanaMasson stain, similar ratio of basal pigmentation was demonstrated. Hyperkeratosis was present in 12 biopsies (40\%). Interface changes and epidermal vacuolization was found in 4 biopsies (13.3\%), all were included in PUVA group of patients.

Dermal infiltrates composed mainly of mononuclear cells (Lympho-histiocytic); seven biopsies (46.7\%) in PUVA group and 7 biopsies (46.7\%) in NB-UVB group showed sparse infiltrate, 7 biopsies (46.7\%) in PUVA group and 5 biopsies (33.3\%) in NB-UVB group showed moderate infiltrate, and 1 biopsy (6.7\%) in PUVA group
Table 4. Side effects reported in the study groups.

\begin{tabular}{ccc}
\hline $\begin{array}{c}\text { NB-UVB group } \\
\mathrm{N}=15\end{array}$ & $\begin{array}{c}\text { PUVA group } \\
\mathrm{N}=15\end{array}$ & Side effects \\
\hline 3 patients (20\%) & 4 patients (26.7\%) & Pruritus \\
1 patient (6.7\%) & $0(0 \%)$ & Photo dermatitis \\
$0(0 \%)$ & 7 patients (46.7\%) & Nausea \\
$0(0 \%)$ & 1 patient (6.7\%) & $\begin{array}{c}\text { Elevation in the liver enzymes } \\
\text { Recurrence at the same } \\
\text { site of treatment }\end{array}$ \\
3 patients (20\%) & 1 patient (6.7\%) & New lesions in other sites \\
\hline patients (26.7\%) & 1 patient (6.7\%)
\end{tabular}

Table 5. Histopathological findings in the center of the vitiliginous lesions before treatment.

\begin{tabular}{|c|c|c|c|c|}
\hline \multirow{2}{*}{\multicolumn{2}{|c|}{ Histopathological changes }} & \multicolumn{3}{|c|}{ Vitiligo center } \\
\hline & & $\begin{array}{c}\text { PUVA } \\
(\mathrm{n}=15)\end{array}$ & $\begin{array}{c}\text { NB-UVB } \\
(\mathrm{n}=15)\end{array}$ & $\begin{array}{c}\text { Total } \\
(\mathrm{n}=30)\end{array}$ \\
\hline \multicolumn{2}{|c|}{ Hyperkeratosis } & $\begin{array}{c}7 \text { biopsies } \\
(46.7 \%)\end{array}$ & $\begin{array}{c}5 \text { biopsies } \\
\text { (33.3\%) }\end{array}$ & $\begin{array}{c}12 \text { biopsies } \\
(40 \%)\end{array}$ \\
\hline \multicolumn{2}{|c|}{ Basal melanization } & $\begin{array}{c}5 \text { biopsies } \\
\text { (33.3\%) }\end{array}$ & $\begin{array}{l}3 \text { biopsies } \\
(20 \%)\end{array}$ & $\begin{array}{c}8 \text { biopsies } \\
(26.7 \%)\end{array}$ \\
\hline \multicolumn{2}{|c|}{ Interface changes } & $\begin{array}{l}4 \text { biopsies } \\
\text { (26.7\%) }\end{array}$ & $0(0 \%)$ & $\begin{array}{c}4 \text { biopsies } \\
\text { (13.3\%) }\end{array}$ \\
\hline \multicolumn{2}{|c|}{ Melanophages } & $\begin{array}{c}7 \text { biopsies } \\
(46.7 \%)\end{array}$ & $\begin{array}{l}4 \text { biopsies } \\
(26.7 \%)\end{array}$ & $\begin{array}{c}11 \text { biopsies } \\
(36.7 \%)\end{array}$ \\
\hline \multicolumn{2}{|c|}{ Eosinophils } & $\begin{array}{c}1 \text { biopsy } \\
(6.7 \%)\end{array}$ & $\begin{array}{c}0 \\
(0 \%)\end{array}$ & $\begin{array}{c}1 \text { biopsies } \\
(3.3 \%)\end{array}$ \\
\hline \multirow{3}{*}{$\begin{array}{l}\text { Lymphohistiocytic } \\
\text { infiltrate }\end{array}$} & Sparse & $\begin{array}{l}7 \text { biopsies } \\
(46.7 \%)\end{array}$ & $\begin{array}{l}7 \text { biopsies } \\
(46.7 \%)\end{array}$ & $\begin{array}{c}14 \text { biopsies } \\
(46.7)\end{array}$ \\
\hline & Moderate & $\begin{array}{l}7 \text { biopsies } \\
(46.7 \%)\end{array}$ & $\begin{array}{l}5 \text { biopsies } \\
\text { (33.3\%) }\end{array}$ & $\begin{array}{c}12 \text { biopsies } \\
(40 \%)\end{array}$ \\
\hline & Dense & $\begin{array}{c}1 \text { biopsy } \\
(6.7 \%)\end{array}$ & $\begin{array}{l}3 \text { biopsies } \\
(20 \%)\end{array}$ & $\begin{array}{c}4 \text { biopsies } \\
(13.3 \%)\end{array}$ \\
\hline
\end{tabular}

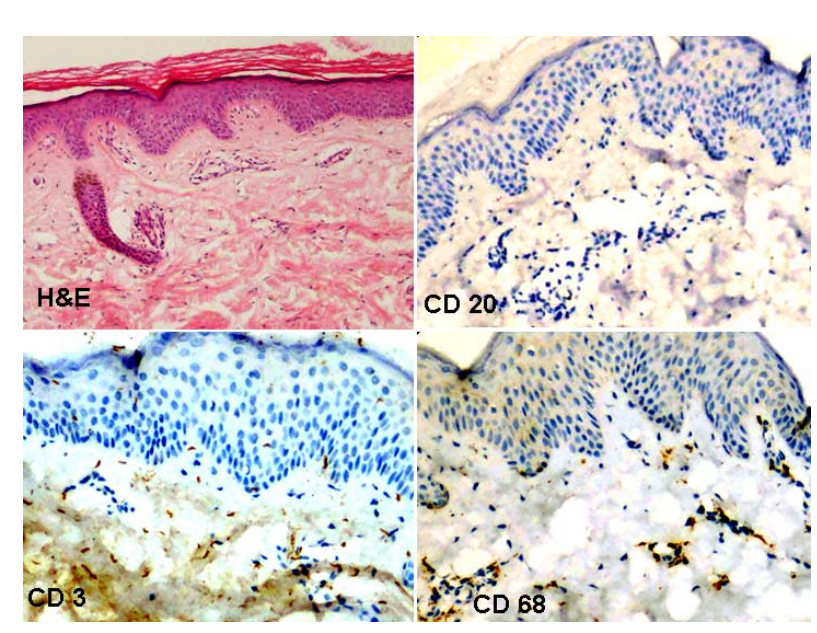

Figure 1. The center of vitiliginous area before treatment; H\&E staining showing almost no basal melanization, hyperkeratosis and sparse superficial dermal lympho-histiocytic infiltrate (upper left, $\times 10$ ). CD20 stained section showing negative lymphocytic staining (upper right, $\times 20$ ). CD3 stained section showing mild positive lymphocytic staining (lower left, $\times 20$ ). CD68 stained section showing strong positive lymphocytic staining (lower right, $\times 20$ ). 
and 3 biopsies (20\%) in NB-UVB group showed a denser infiltrate. Melanophages appeared in the upper dermis in 7 biopsies (46.7\%) in PUVA group and in 4 biopsies (26.7\%) in NB-UVB group (Figure 2). Eosinophils appeared in the infiltrate in only 1 biopsy (6.7\%) in PUVA group.

After treatment (Table 6, Figures 3 and 4), 29 biopsies were obtained from the repigmented areas (14 in PUVA group and 15 in NB-UVB group), and another 24 biopsies were obtained from the non-responding depigmented areas of the vitiliginous lesions (14 in PUVA group and 10 in NB-UVB group). The remaining 5 improved patients in NB-UVB group showed complete repigmentation with no non-pigmented islands to obtain biopsies.

The non-responding areas after treatment showed no basal melanization in 21 biopsies (87.5\%) of 24 biopsies and the presence of basal melanization in 3 biopsies (12.5\%) (1 of 14 biopsies in PUVA group and 2 of 10 biopsies in NB-UVB group) while the repigmented areas of the vitiliginous lesions showed the presence of basal melanization in all 29 biopsies (100\%). Melanin was detected using Fontana Masson stain in same ratio of biopsies. Hyperkeratosis was present in 3 biopsies $(12.5 \%)$ of the non-responding areas (1 biopsy in PUVA group and 2 biopsies in NB-UVB group) and was present in 2 biopsies $(6.89 \%)$ of 29 biopsies of the repigmented areas (1 biopsy in PUVA group and in 1 biopsy in NB-UVB group).

Interface changes and epidermal vacuolization were more manifested in biopsies obtained from cases treated with NB-UVB than in biopsies obtained from cases treated with PUVA (Figures 3 and 4 ) and were found in 3 biopsies (12.5\%) of the non-responding areas (1 biopsy in PUVA group and 2 biopsies in NB-UVB group) and were found in 11 biopsies (37.9\%) of the repigmented areas (4 biopsies in PUVA group and 7 biopsies in NBUVB group).
Sparse infiltrate was present in 14 biopsies (58.33\%); 11 biopsies (78.57\%) in PUVA group and 3 biopsies (20\%) in NB-UVB group, moderate infiltrate was present in 8 biopsies (33.34\%); 3 biopsies (21.43\%) in PUVA group, and in 5 biopsies (50\%) in NB-UVB group and dense infiltrate was present in 2 biopsies (8.33\%) in NBUVB group only.

Melanophages appeared in 7 biopsies (29.16\%) of 24 biopsies; 4 biopsies (28.6\%) in PUVA group and in 3 biopsies (30\%) in NB-UVB group. Eosinophils appeared in the infiltrate in 4 biopsies (16.66\%) in PUVA group only.

Regarding the repigmented areas of the vitiliginous lesions; sparse infiltrate was present in 10 biopsies (34.48\%) of 29 biopsies; 7 biopsies (50\%) in PUVA group and 3 biopsies (30\%) in NB-UVB group, moderate infiltrate was present in 16 biopsies (55.17\%); 7 biopsies (50\%) in PUVA group and 9 biopsies (60\%) in NB-UVB group and dense infiltrate was present in 3 biopsies (20\%) in NB-UVB group only.

Melanophages appeared in 22 biopsies (75.8\%) of 29 biopsies; 10 biopsies (71.43\%) in PUVA group and in 12 biopsies (80\%) in NB-UVB group.

Interface changes appeared in 11 biopsies (37.9\%); 4 biopsies (28.6\%) in PUVA group and 7 biopsies (46.7\%) in NB-UVB group.

Using Fontana-Masson staining melanin was detected in all 29 biopsies (100\%) of the repigmented areas after treatment (Figure 4).

Immunohistochemical findings using MART-1 antibody; biopsies from the center of the vitiliginous area before treatment showed:

MART-1 negative basal staining (Figure 2) was demonstrated in 22 biopsies (73.3\%) of 30 and the presence of MART-1 positive cells in 8 biopsies (26.7\%) out of 30 biopsies taken from center of the vitiliginous lesions.

Biopsies from the non-responding areas after treatment

Table 6. Histopathological changes after treatment in the non-responding and repigmented areas of the vitiliginous lesions.

\begin{tabular}{|c|c|c|c|c|c|c|}
\hline \multirow{2}{*}{ Histopathological changes } & \multicolumn{3}{|c|}{ Non-responding areas } & \multicolumn{3}{|c|}{ Repigmented areas } \\
\hline & PUVA $(\mathrm{n}=14)$ & NB-UVB $(n=10)$ & Total $(n=24)$ & PUVA $(n=14)$ & NB-UVB ( $\mathrm{n}=15)$ & Total $(\mathrm{n}=29)$ \\
\hline Basal melanization & 1 biopsy (7.14\%) & 2 biopsies (20\%) & 3 biopsies (12.5\%) & 14 biopsies (100\%) & 15 biopsies (100\%) & 29 biopsies (100\%) \\
\hline Interface changes & 1 biopsy (7.14\%) & 2 biopsies (20\%) & 3 biopsies (12.5\%) & 4 biopsies (28.6\%) & 7 biopsies (46.7\%) & 11 biopsies (37.9\%) \\
\hline Melanophages & 4 biopsies (28.6\%) & 3 biopsies (30\%) & 7 biopsies (29.16\%) & $\begin{array}{c}10 \text { biopsies } \\
(71.43 \%)\end{array}$ & 12 biopsies (80\%) & 22 biopsies (75.8\%) \\
\hline Eosinophils & 4 biopsies $(28.6 \%)$ & $0(0 \%)$ & 4 biopsies (16.66\%) & $0(0 \%)$ & $0(0 \%)$ & $0(0 \%)$ \\
\hline \multirow{3}{*}{$\begin{array}{l}\text { Lymphohistiocytic } \\
\text { infiltrate: }\end{array}$} & $\begin{array}{c}11 \text { biopsies } \\
(78.57 \%)\end{array}$ & 3 biopsies (30\%) & 14 biopsies ( $58.33 \%)$ & 7 biopsies (50\%) & 3 biopsies (20\%) & 10 biopsies (34.48\%) \\
\hline & 3 biopsies (21.43\%) & 5 biopsies (50\%) & 8 biopsies (33.34\%) & 7 biopsies (50\%) & 9 biopsies $(60 \%)$ & 16biopsies (55.17\%) \\
\hline & $0(0 \%)$ & 2 biopsies (20\%) & 2 biopsies (8.33\%) & $0(0 \%)$ & 3 biopsies (20\%) & 3 biopsies (10.34\%) \\
\hline
\end{tabular}




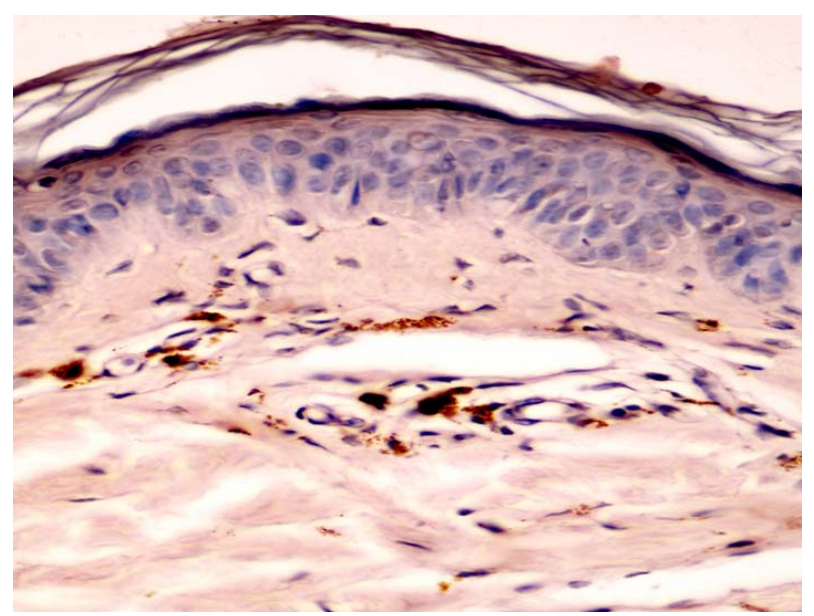

Figure 2. MART-1 immune-staining of vitiligo center showing no basal melanization with considerable melanophages in the dermis immunologically stained $(\times 40)$.

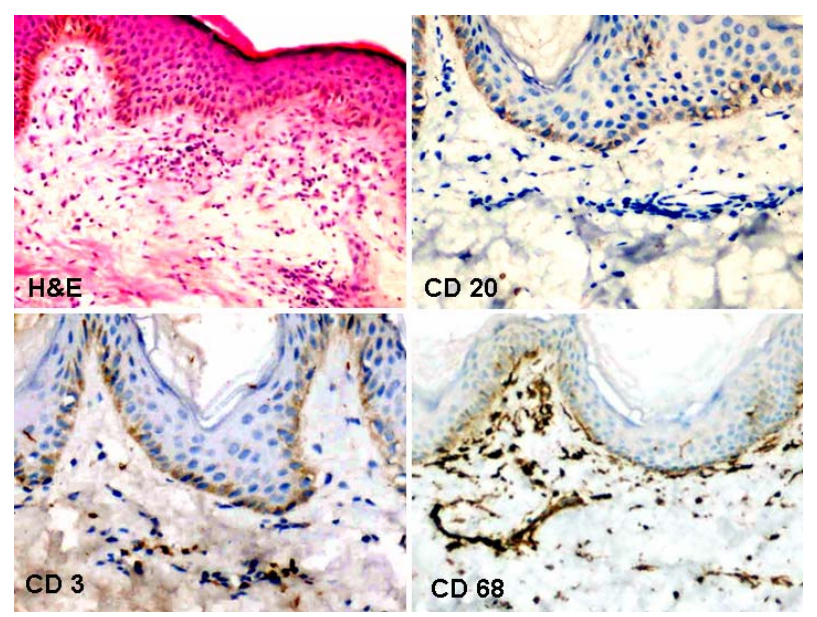

Figure 3. The repigmented area after treatment with NBUVB ( $\times 20):$ H\&E staining showing basal melanization and moderate upper dermal lympho-histiocytic infiltrate with interface activity (upper left). CD20 stained section showing negative lymphocytic staining (upper right). CD3 stained section showing mild positive lymphocytic staining (lower left). CD68 stained section showing very strong positive lymphocytic staining (lower right).

showed MART-1 negative staining in all biopsies except for 3 biopsies (12.5\%).

Biopsies from repigmented areas after treatment showed presence of MART-1 positive cells in all 29 biopsies (100\%) taken from repigmented areas after treatment with PUVA and NB-UVB (Figure 4). MART-1 stain also demonstrated the striking presence of melanophages in the dermis (Figure 2).

Immunohistochemical findings (Figures 1 and 3) revealed that CD68-positive cells were the most abundant cells in all biopsies, i.e. the vitiliginous areas before treatment, the non-responding areas after treatment and the repigmented areas after treatment. CD3-positive cells were

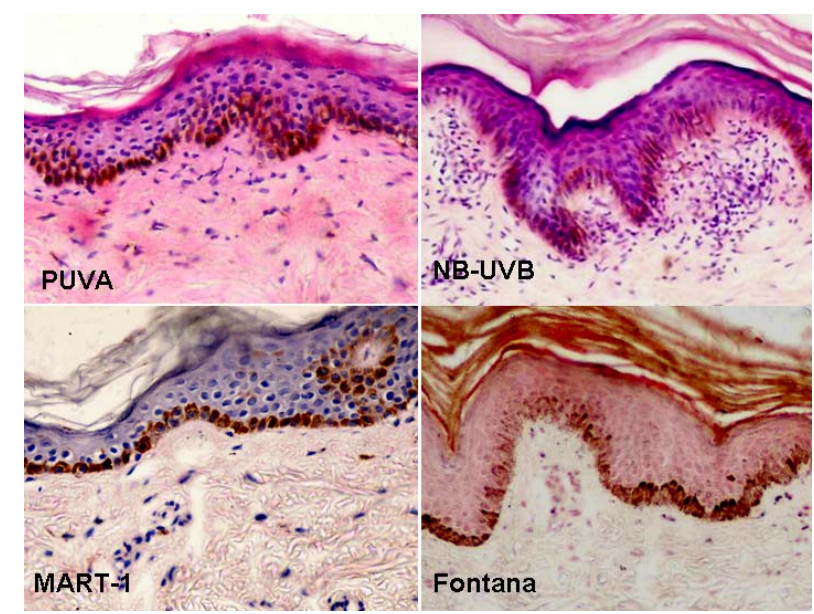

Figure 4. The repigmented area after treatment $(\times 20)$ : After PUVA; H\&E stained section showing basal melanization and sparse superficial dermal lympho-histiocytic infiltrate (Upper left). After NB-UVB; H\&E stained section showing basal melanization and moderately dense dermal lymphohistiocytic infiltrate with interface activity (upper right). MART-1 immune-stained section after PUVA treatment showing positive basal staining (lower left). Fontana-Masson staining after treatment showing the presence of basal melanization (lower right).

also detected in similar biopsies, but CD3-positive cells were less abundant than the CD68-positive cells. No significant CD20-positive cells were observed in all biopsies. These findings demonstrated the striking presence of histiocytes besides T-lymphocytes in the dermal infiltrate.

\section{Discussion}

Phototherapy is one of the most commonly used treatments for vitiligo with appreciated success rates and low incidence of side effects. It is also highly recommended after surgical grafting treatments to guarantee success [10].

Oral PUVA is the combined use of a photosensitizing chemical compound and non-ionizing electromagnetic radiation, and this combination induces a beneficial result not produced by either alone [6]. NB-UVB is a more recent phototherapy modality which was developed to remove the shorter erythemogenic wavelengths and to use a concentrated UVB source of $311 \mathrm{~nm}$. NB-UVB has been reported to be effective and safe in vitiligo [11].

In this study we tried to have clinical objective evaluation of improvement after phototherapy by using a planimeter to calculate percentage of improvement and categorize the resulting numbers into descriptive terms for overall evaluation.

Clinical evaluation showed that only 1 patient (3.3\%) failed to respond to therapy (PUVA group) while the remaining 29 patients (93.3\%) demonstrated improvement 
ranging from excellent to mild repigmentation. The present study demonstrated that NB-UVB group patients showed statistically significant better results than oral PUVA group patients. Similar success rates were obtained in previous comparative studies demonstrating superiority of NB-UVB to PUVA [7,12]. In our study we also demonstrated the faster response to NB-UVB which provided considerable repigmentation (up to 90\%) after only 2 months of therapy compared to mild improvement (up to 37\%) in the PUVA group after same period.

Another dramatic finding was the difference in the color matching between the two modalities of treatment, which was excellent in all patients treated with NB-UVB while $42.8 \%$ of patients treated with oral PUVA showed darker unsightly repigmentation of the treated vitiliginous areas compared to the patient's unaffected skin. This hyperpigmentation has been reported previously too [7]. The predominance of perifollicular repigmentation as a main pattern of repigmentation after both modalities was clearly demonstrated.

The starting doses of NB-UVB in previous studies varied considerably, ranging from $75 \mathrm{~mJ} / \mathrm{cm}$ [13] to 740 $\mathrm{mJ} / \mathrm{cm}$ [12], although these differences may reflect variations in calibration. Some studies used starting doses based on the minimum erythema dose [14]. In other reported studies the starting dose was based on skin typing [15]. In the present study, the starting dose was 0.574 $\mathrm{J} / \mathrm{cm}^{2}$ based on patients' main skin type (type 4). The therapeutic dose ranged from 1.402 to $2.737 \mathrm{~J} / \mathrm{cm}^{2}$.

The first exposure dose for PUVA treated patients in previous reported studies was based on minimal phototoxic dose (MPD) to ensure a safe starting dose [16]. In other reported studies, the starting dose was based on skin typing [17]. In the present study the starting dose was $2 \mathrm{~J} / \mathrm{cm}^{2}$ based on patients' main skin type. The therapeutic dose ranged between 3.906 and $6.104 \mathrm{~J} / \mathrm{cm}^{2}$. In other previous reported studies, the therapeutic dose did not exceed $5 \mathrm{~J} / \mathrm{cm}^{2}$ for PUVA and $2 \mathrm{~J} / \mathrm{cm}^{2}$ for NB-UVB [7]. The higher therapeutic doses utilized here may be correlated to the darker skin type and skin tolerance to radiation in our locality due to intensive sun exposure throughout the year.

The PUVA patients were older than the NB-UVB patients due to the exclusion criteria used in selecting its patients. Age was not found to influence the outcome of vitiligo therapy with oral PUVA and NB-UVB in previous studies [18]. However, the presumption that the earlier the patient was treated, the better was the response also exists [19] and we agree that the age factor cannot be denied when comparing results in vitiligo cases.

Oral PUVA is not recommended for children younger than 12 years $[4,6]$. The phototoxic potential of psoralen is of great concern in children where avoidance of excessive sun exposure may be difficult to regulate, again, the risk of carcinogenesis and premature aging of skin cannot be neglected in children with long-term use. On the other hand, NB-UVB has been reported to be effective and safe in childhood vitiligo [11]. In the present study, 8 (80\%) out of 10 children developed more than $75 \%$ overall repigmentation after 4 months of NB-UVB therapy. Thus, higher success rates were observed with NBUVB radiation therapy.

NB-UVB therapy offers other advantages over oral PUVA therapy that makes it preferable for most patients. In particular, PUVA therapy requires the use of eye protection after treatment sessions, cannot be used in pregnancy, is contraindicated in patients with hepatic impairment or who are taking warfarin or phenytoin, and requires the somewhat inconvenient prior administration of psoralen. Of greatest concern however, is the potential of PUVA to cause non-melanoma and melanoma skin cancer [20], although in our vitiligo patients this was not detected at all for several years of UV therapy (data not shown), perhaps because of the skin type and genetic background in our locality. Nevertheless, it is important to mention that NB-UVB seems to be considerably safer than PUVA in required number of treatment sessions as higher success rates can be achieved as early as 2 months of therapy. Although risks for cutaneous malignancies are reported with PUVA, however, to date there is insufficient data available to provide recommendation regarding a safe maximum narrow band UVB dose.

The general histological picture of vitiligo before treatment revealed the presence of hyperkeratosis in $40 \%$ of vitiligo skin biopsies. This finding was noticed in previous studies [21]. Hyperkeratosis declined after treatment, down to $12.5 \%$ of the non-responding areas and in $6.89 \%$ of the repigmented areas (with no significant difference between the 2 study groups). The presence of hyperkeratosis in a lower ratio after treatment may be due to the return of the epidermal turn over rate due to the stoppage of the keratinocytes degeneration and death process.

Basal melanization was detected in vitiligo skin in 26.6\%. Kim et al. (2008) found remaining melanocytes in $12 \%$ vitiligo skin cases by using MART-1 stain. In the present study, using MART-1 antibody for immunohistochemical staining of melanocytes, MART-1 positive cells were found in the vitiligo skin in a higher percentages than in previous studies. After 4 months of phototherapy treatment, basal melanization appeared in all biopsies of the repigmented areas and was present in $12.5 \%$ of the non-responding areas of the vitiliginous lesions after treatment.

The present study demonstrated the presence of moderate to dense perivascular and perifollicular lymphohistiocytic infiltrate in $53.3 \%$ of vitiliginous skin. This existence of the inflammatory infiltrate in the vitiliginous 
area confirms the role of the immunological inflammatory process in initiation of the disease, which was described in previous literature [22]. After treatment, biopsies from the non-responding areas of the vitiliginous lesions treated with NB-UVB showed the presence of a moderate to dense lympho-histiocytic infiltrate in $70 \%$ of biopsies compared with $21.43 \%$ of biopsies taken from the non-responding areas of the vitiliginous lesions treated with PUVA. Biopsies from the repigmented areas after treatment with NB-UVB showed moderate to dense dermal lympho-histiocytic infiltrate in $80 \%$ of biopsies compared to $50 \%$ of cases treated with PUVA. The overall density was higher in NB-UVB treated cases.

Comparison of the responding pigmented islands and the non responding depigmented spots; it was obvious that lympho-histiocytic infiltrates, interface changes and melanophages were in significant higher levels in healed areas while in low levels in the non repigmented islands. These pathological findings seem strictly correlating to existence of melanocytes and melanin in the epidermis which represents the targeted antigen in such cases (Table 6).

All sections before treatment did not show staining for CD20 confirming that the B lymphocytes do not have a main role in the pathogenesis of vitiligo while CD3 staining was positive in most sections demonstrated that $\mathrm{T}$ lymphocytes play a major role in the disease pathogenesis. Staining cells positively again with CD68 demonstrated that histiocytes and macrophages have a main bulk in the infiltrate.

After treatment, similar staining for CD3 and CD68 with absence of CD20-positive cells was well demonstrated. Staining for CD3 \& CD68 was correlated to lympho-histiocytic density in H\&E stained sections with higher ratio in NB-UVB treated group.

Interface changes and epidermal vacuolization appeared in the present study in $13.3 \%$ of pretreated vitiligo skin. Epidermal vacuolization occurs as a result of the interface changes where the dermal lympho-histiocytic cells attack the melanotic basal cells leading to cell degeneration and epidermal vacuolization. After treatment, an increase in interface changes and basal vacuolization was found in both the non-responding and the repigmented areas of NB-UVB cases more than the non-responding and the repigmented areas of PUVA cases. Significant higher levels of interface changes and vacuolization in NB-UVB treated patients were correlating to the density of lympho-histiocytic infiltration.

$\mathrm{H} \& \mathrm{E}$ and MART-1 stains revealed the presence of considerable dermal melanophages in $36.6 \%$ of vitiligo skin before and also after treatment. Other previous studies revealed the presence of melanophages but in lower ratios [21]. Ackerman et al. (1997) reported that the presence of melanophages in the papillary dermis is a helpful clue to exclude vitiligo from the differential diagnoses of a hypopigmented or depigmented lesion [23]. The striking presence of melanophages in vitiligo in the present study contradicts that view. The higher percentages of melanophages could be related to the darker skin type of the patients with epidermis containing higher melanin levels which after the interface activity and basal vacuolization drop into dermis to be targeted by histiocytes and macrophages. More melanophages were demonstrated in biopsies of repigmented areas in both treated groups due to the higher epidermal melanin contents and the attacking infiltrates in the dermis.

The more dense lympho-histiocytic infiltrates and more interface changes with basal vacuolization findings in NB-UVB cases may correlate with the higher recurrence and reactivation ratio of vitiligo in such patients. Thus, a more potent immunological suppression by PUVA over NB-UVB is demonstrated and could explain higher recurrence and development of new lesions in NB-UVB group of patients (NB-UVB group 46.7\%, PUVA group 13.4\%). Therapeutic effect of PUVA in vitiligo was related to selective cytotoxicity and apoptosis of inflammatory cells, apart from its ability to induce melanocytes migration to the epidermis from the outer root sheath of the hair through the action of immune cytokines and inflammatory mediators released [24]. Lymphocytic suppression by PUVA therapy is well documented, offering an explanation for the clinical response that PUVA produces in other lymphocyte-rich skin diseases such as psoriasis [25].

Vitiligo is an unstable disease and its activity is usually unpredicted but related to immunological stimulation and subsequent destruction of new melanocytes. Detecting active cases after both modalities needed to be done in a further study to confirm further advantage of a method to another, although we expect that PUVA will be superior in such cases due to its ability to reduce immunological inflammatory infiltrates with subsequent clinical decline in disease activity and less development of new lesions as described previously in the results (Figure 4, Tables 3 and 4).

\section{Conclusions}

In conclusion, NB-UVB treatment can provide statistically significant better results than PUVA. Distinct advantages over PUVA include the lack of psoralen-related side-effects and precautions, a cosmetically better color match, and safety in children. Although NB-UVB therapy gives a more rapid effect than oral PUVA therapy, yet PUVA therapy was accompanied by significant less recurrence and less development of new vitiligo lesions.

The dermal infiltrates, composed mainly of T cells and Histiocytes, with the interface changes persisted after the 
NB-UVB repigmentation more than in the PUVA treated areas. Thus, PUVA may provide better immuno-modulatory effect on vitiligo than NB-UVB and may give better response regarding the disease reactivation and recurrence. A combination of UVA and NB-UVB bulbs in same treating cabinets maybe recommended obtaining better treatment results.

\section{REFERENCES}

[1] S. O. Kovacs, "Vitiligo," Journal of the American Academy of Dermatology, Vol. 38, No. 5, 1998, pp. 647-666.

[2] M. D. Njoo, W. Westerhof, J. D. Bos and P. M. Bossuyt, "The Development of Guidelines for the Treatment of Vitiligo," Archives of Dermatology, Vol. 135, No. 12, 1999, pp. 1514-1521.

http://dx.doi.org/10.1001/archderm.135.12.1514

[3] T. M. Runger, "Ultraviolet Light,” In: J. L. Bolognia, J. L. Jorizzo, R. P. Rapini, T. D. Horn, A. J. Maoncini, J. M. Mascaro, S. J. Salasche, J. H. Saurat and G. Stingl, Eds., Dermatology, Mosby, Edinburgh, London, New York, Oxford, Philadelphia, St. Louis, Sydney, Toronto, Vol. 1, 2003, pp. 1353-1364.

[4] M. El-Mofty and H. El-Sawaalhy, "Clinical Study of a New Preparation of 8-Methoxypsoralen in Photochemotherapy," International Journal of Dermatology, Vol. 33, No. 8, 1994, pp. 688-703.

http://dx.doi.org/10.1111/j.1365-4362.1994.tb02904.x

[5] D. Parsad, A. J. Kanwar and B. Kumar, "Psoralen-Ultraviolet A vs. Narrowband Ultraviolet B Phototherapy for the Treatment of Vitiligo," Journal of the European Academy of Dermatology and Venereology, Vol. 20, No. 2, 2006, pp. 175-177.

http://dx.doi.org/10.1111/j.1468-3083.2006.01413.x

[6] A. K .Gupta and T. F. Anderson, "Psoralen Photochemotherapy," Journal of the American Academy of Dermatology, Vol. 17, No. 5, 1987, pp. 703-734.

http://dx.doi.org/10.1016/S0190-9622(87)70255-2

[7] S. S. Yones, D. Der, A. Roy, et al., "Efficacy of PsoralenUV-A Therapy vs Narrowband-UV-B Therapy," Archives of Dermatology, Vol. 143, No. 5, 2007, pp. 578-584. http://dx.doi.org/10.1001/archderm.143.5.578

[8] R. S. Dawe, J. Ferguson and S. H. Ibbotson, "A Study of the Characteristics of Erythema Induced by Bath and Oral Methoxsalen Photochemotherapy and Narrowband Ultraviolet," Journal of Photochemistry and Photobiology, Vol. 78, No. 1, 2003, pp. 55-60.

http://dx.doi.org/10.1562/0031-8655(2003)078<0055:AIS OTC>2.0.CO;2

[9] Y. Kawakami, K. Jane, K. Takeshi, et al., "Production of Recombinant MART-1 Protiens and Specific AntiMART1 Polyclonal and Monoclonal Antibodies: Use in the Characterization of the Human Melanoma Antigen MART1," Journal of Immunological Methods, Vol. 202, No. 1, 1997, pp. 13-25.

http://dx.doi.org/10.1016/S0022-1759(96)00211-6

[10] S. S. Awad, H. Abdel-Raof, W. Hosam El-Din and M. El-Domyati, "Epithelial Grafting for Vitiligo Requires
Ultraviolet A Phototherapy to Increase Success Rate,” Journal of Cosmetic Dermatology, Vol. 6, No. 2, 2007, pp. 119-124.

http://dx.doi.org/10.1111/j.1473-2165.2007.00307.x

[11] M. D. Njoo, J. D. Bos and W. Westerhof, "Treatment of Generalized Vitiligo in Children with Narrow-Band (TL01) UVB Radiation Therapy," Journal of the American Academy of Dermatology, Vol. 42, No. 2, 2000, pp. 245253. http://dx.doi.org/10.1016/S0190-9622(00)90133-6

[12] M. El Mofty, W. Mostafa, S. Esmat, et al., "Narrow Band Ultraviolet B $311 \mathrm{~nm}$ in the Treatment of Vitiligo: Two Right-Left Comparison Studies," Photodermatology, Photoimmunology \& Photomedicine, Vol. 22, No. 1, 2006, pp. 6-11. http://dx.doi.org/10.1111/j.1600-0781.2006.00189.x

[13] W. Westerhof and L. Nieuweboer-Krobotova, "Treatment of Vitiligo with UV-B Radiation vs Topical Psoralen Plus UV-A," Archives of Dermatology, Vol. 133, No. 12, 1997, pp. 1525-1528.

http://dx.doi.org/10.1001/archderm.1997.03890480045006

[14] I. Hamzavi, H. Jain, D. McLean, et al., "Parametric Modeling of Narrowband UV-B Phototherapy for Vitiligo Using a Novel Quantitative Tool," Archives of Dermatology, Vol. 140, No. 6, 2004, pp. 677-683.

http://dx.doi.org/10.1001/archderm.140.6.677

[15] A. Simin, S. Sedef, B. Gonca and K. Fikret, "No Additional Effect of Topical Calcipotriol on Narrow Band UVB Phototherapy in Patients with Generalized Vitiligo," Photodermatology, Photoimmunology \& Photomedicine, Vol. 21, No. 2, 2005, pp. 79-83.

http://dx.doi.org/10.1111/j.1600-0781.2005.00139.x

[16] S. M. Halpern, A. Anstey and R. S. Dawe, "Guidelines for Topical PUVA: A Report of a Workshop of the British Photodermatology Group," British Journal of Dermatology, Vol. 142, No. 1, 2000, pp. 22-31. http://dx.doi.org/10.1046/j.1365-2133.2000.03237.x

[17] S. Valkova, M. Trashlieva and P. Christova, "Treatment of Vitiligo with Local Khellin and UVA: Comparison with Systemic PUVA," Clinical and Experimental Dermatology, Vol. 29, No. 2, 2004, pp. 180-184. http://dx.doi.org/10.1111/j.1365-2230.2004.01462.x

[18] S. Sahin, U. Hindioglu and A. Karaduman, "PUVA Treatment of Vitiligo: A Retrospective Study of Turikish Patients," International Journal of Dermatology, Vol. 38, No. 7, 1999, pp. 542-545. http://dx.doi.org/10.1046/j.1365-4362.1999.00654.x

[19] T. S. Anbar, W. Westerhof, A. T. Abdel-Rahman and M. A. El-Khayyat, "Evaluation of the Effects of NB-UVB in Both Segmental and Non-Segmental Vitiligo Affecting Different Body Sites," Photodermatology, Photoimmunology \& Photomedicine, Vol. 22, No. 3, 2006, pp. 157163. http://dx.doi.org/10.1111/j.1600-0781.2006.00222.x

[20] R. S. Stern, "The Risk of Melanoma in Association with Long-Term Exposure to PUVA," Journal of the American Academy of Dermatology, Vol. 44, No. 5, 2001, pp. 755-761. http://dx.doi.org/10.1067/mjd.2001.114576

[21] Y. C. Kim, H. Y. Kang, S. Seonghyang and E. S. Lee, "Histopathologic Features in Vitiligo," The American Journal of Dermatopathology, Vol. 30, No. 2, 2008, pp. 112-115. 
http://dx.doi.org/10.1097/DAD.0b013e3181651511

[22] L. F. Montes, J. Abulafia, W. H. Wilborn, et al., "Value of Histopathology in Vitiligo," International Journal of Dermatology, Vol. 42, No. 1, 2003, pp. 57-61. http://dx.doi.org/10.1046/j.1365-4362.2003.01743.x

[23] A. B. Ackerman, N. Chongchitant, J. Sanchez, Y. Guo, B. Bennin, M. Reichel and A. B. Randall, "Embryologic, Histologic and Anatomic Ascpects,” In: A. B. Ackerman, N. Chongchitant, J. Sanchez, Y. Guo, B. Bennin, M. Reichel and A. B. Randall, Eds., Histologic Diagnosis of Inflammatory Skin Diseases, 2nd Edition, Williams and Wil- kins, Baltimore, Phildelphia, London, Paris, Banckok, Hong Kong, Sydney, Tokyo, 1997, p. 2.

[24] T. B. Fitzpatrick, "Mechanisms of Phototherapy of Vitiligo," Archives of Dermatology, Vol. 133, No. 12, 1997, pp. 1591-1592.

http://dx.doi.org/10.1001/archderm.1997.03890480113020

[25] R. M. Halder and C. M. Young, "New and Emerging Therapies for Vitiligo,” Dermatologic Clinics, Vol. 18, No. 1 , 2000, pp. 79-83.

http://dx.doi.org/10.1016/S0733-8635(05)70149-2 